\title{
COGITO in Space
}

\author{
Daniela de Paulis \\ Artist in Residence, Dwingeloo Radio \\ Telescope \\ Rotterdam, The Netherlands \\ selavyrose@gmail.com
}

\section{INTRODUCTION}

COGITO in Space is an interdisciplinary art project speculating on the creative and philosophical possibilities of exploring the cosmos by means of radio waves. The project allows participants to send their brain activity into space, as a symbolic action for shifting awareness from an Earth-centred perspective to a cosmos-wide perspective.

COGITO in Space has been developed in collaboration with Frank White, author of The Overview Effect: Space Exploration and Human Evolution, a pivotal book that explores the shift in worldview experienced by astronauts when they see the Earth from space and in space.

\section{CONCEPTUAL BACKGROUND OF THE PROJECT}

COGITO in Space is an experiential narrative sending thoughts into outer space as radio waves. The project exists both as a mobile installation and as performative event staged inside the cabin of the Dwingeloo radio telescope in The Netherlands. For both versions of the project, a team composed by three neuroscientists prepare the subject with a lab grade electroencephalogram (EEG) device and a virtual reality (VR) headset, showing an experimental video of the Earth seen from space. The brain activity stimulated by the video is recorded and simultaneously transmitted into space in real time, using the antenna of the Dwingeloo radio telescope.

COGITO in Space is an interdisciplinary, collaborative project and the result of six years research at the Dwingeloo radio telescope and ASTRON (the Dutch Institute for Radio Astronomy). The intellectual background of the project grew over the years, thanks to the contribution of specialists from different fields, however my reasoning behind it focuses mainly on two concepts: the unresolved question of Mind and
Body Dualism and the Overview Effect. Another important reference was the novel Solaris by Stanislaw Lem and the homonymous film by Andrei Tarkovsky.

The reasoning around the project began as a reflection upon the use of the electromagnetic spectrum, and especially radio waves, by radio astronomers for gathering data of cosmic phenomena, and upon the type of knowledge we gain from the discovery of such remote events that cannot be known through direct sensory experience. Working alongside radio astronomers over the years, I had the opportunity to better understand the relevance of radio transmissions in contemporary thought and their great cultural impact.

My questioning focused especially on how radio waves transmissions have been expanding the human reach into the cosmos, towards places where manned space exploration might never be able to reach, allowing us to remotely explore outer space and exposing the conventional perception of our surroundings to a virtual yet uncannily real and detailed landscape, made of matter still to be defined. Radio waves have gradually become the mean for the virtual human space travel and the carriers of a newfound cosmic awareness and cultural contents, exceeding their scientific and technological function.

One of the questions that originated from my research, and that eventually became one of the leading concepts of COGITO in Space, is "how does the knowledge acquired through remote observation of the universe influence our cognition and how does the mind interact with the matter of such distant universe and vice versa?" (de Paulis 2017, p.178) 


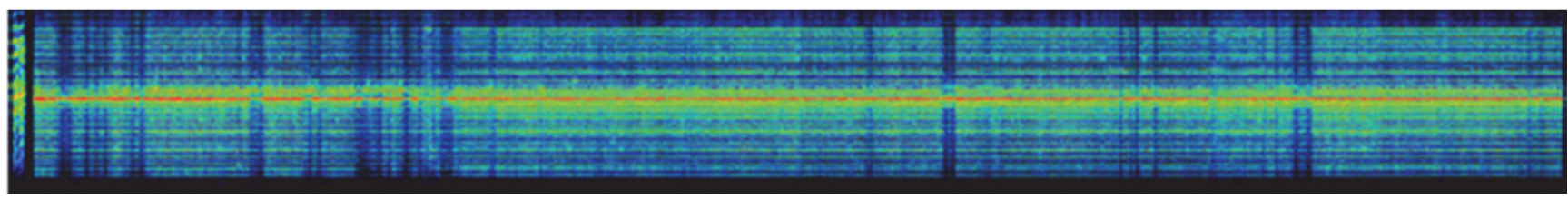

Figure 1: Brain activity recording during COGITO on the 5 November 2018 (credit: Michael Sanders).

\section{PERFORMANCE AT THE DWINGELOO RADIO TELESCOPE}

On the 5 November 2018, COGITO in Space settled at the Dwingeloo radio telescope, its originally intended physical location. The project was designed from the start to be presented inside the cabin of a radio telescope that would mimic the post-human outer shell of the body, its life support system in a half human-half technological system.

The Dwingeloo radio telescope was inaugurated in 1956 and established as part of the Dutch heritage in 2011. The cabin still features some of the original radio equipment, together with modern amateur radio devices and its environment has become the iconic filmic set for the on-going project's performances since 2014.

I conceived the event at the radio telescope to be an experience for the visitors rather than a conventional exhibition opening, with several activities taking place throughout the day, in form of cinematic reality for which the extra-ordinary and ordinary mixed. The day started at the ASTRON auditorium with a symposium and keynote lectures by space philosopher Frank White, cultural anthropologist Fred Spier, retired NASA astronaut Nicole Stott and moderated by art critic Josephine Bosma. Frank White introduced the Overview Effect and his more recent concept of Cosma Hypothesis, suggesting that while exploring outer space, humankind should avoid repeating the mistakes committed during colonial explorations of Earth.

Fred Spier is professor of Big History at the University of Amsterdam. Big History is a cuttingedge field of research investigating the development of both natural and cultural events, from the Big Bang to the present, working along a very broad time scale from an interdisciplinary perspective, merging human sciences with physics, astronomy and geology. Spier spoke about his experience of the Moon landing as a child and the cultural meaning of the Earth Rise photo, which for many years had a strong impact only on the American narrative, leaving Europe and the rest of the world almost indifferent. Astronaut Nicole Stott spoke on Skype about her personal experience of the Overview Effect from the International Space
Station and her life on board, together with her crew mates.

The symposium was followed by a conversation between myself, the neuroscientists, and Josephine Bosma, who asked about my background as a contemporary dancer and how that affected the making of COGITO in Space. In my answer it emerged that the physical division between oneself and outer space is a perceptive illusion, as matter at the atomic level is a continuity, the difference between one's body and outer space being an instance of intensity and density of matter: the blood running through my veins as I write is, in fact, as close to my skin - the body's membrane between interior and exterior space - as the air and materials touching it. My internal organs are, thus, a continuity with outer space.

In COGITO in Space the body streams beyond its protective skin into the infinite space beyond it, poetically drifting towards the unknown. The talks session was followed by a meditative lecture-walk in the area surrounding the scientific facilities, the Dwingeldverveld National Park, mostly a flat landscape dotted with heath lands. The walk, guided by planetary scientist Maarten Roos, took the visitors along a straight line going from the radio telescope into the wide open landscape and back, allowing for the visitors to gaze as far as the horizon, as if viewing the curvature of the Earth.

The walk, lasting approximately one hour, was interspersed by cogitations on the origin of the cosmos, of life on Earth, the existence of possible extraterrestrial life, and was informed by Fred Spier's book Big History and the Future of Humanity. The walk aimed at inspiring a sense of belonging to Earth before virtually leaving the planet, as well as creating room for individual existential questioning. It was followed by an introduction into the history of radio astronomy and the Dwingeloo radio telescope by astronomer Roy Smits. The short presentation led the visitors inside the cabin through a seamless transition between the different events taking place and the different people performing them.

While the visitors started entering the cabin and taking their seats, I turned the engine of the radio telescope on, while the participant lying on a gravity 
chair was being prepared by the neuroscientists with the EEG device and the VR headset. As the people sat still, the radio telescope started tracking the star Betelgeuse, the departing point for the brain activity transmission, and this triggered the rotation of the cabin. Once reached the complete stop of the rotation, the curtains lowered, shortly after the video in virtual reality started playing while the brain activity was recorded and simultaneously I started transmitting the signal into space, with the antenna of the radio telescope pointing still towards the sky. This action allowed for the brain activity to be spread across a large portion of the sky with the Earth's rotation.

Before initiating the radio transmissions, I pronounced through the transmitter the call sign of the Dwingeloo radio telescope, PI9CAM - Papa India Nine Charlie Alpha Mike - the identifier of the radio station used, a standard procedure in amateur radio.

The instantaneous visualization of the brain activity recorded was projected inside the cabin together with the visualization of the radio transmission happening simultaneously. The sound produced by the brain activity created hypnotic and repetitive patterns that generated a meditative mood inside the cabin: people experiencing the event seemed to draw their attention inwards and join the participant in her intimate journey with the mind in outer space. The performance ended with me speaking the call sign through the transmitter and resuming the radio transmission. The entire performance lasted approximately forty-five minutes and was repeated twice during the day.

Following the event on the $5_{\text {th }}$ of November, participants contacted me reporting to have lived a special day and that the experience will remain in their memory as a unique moment. The performance used a purposefully objective and abstract language, assimilating the event to a scientific presentation, my intention being to allow for the experience to find its niche in the individual memory according to one's inner understanding, regardless of the specialist aesthetic appreciation by the participant and the observer. The work in progress and the event at the Dwingeloo radio telescope have been documented as part of a reportage filmed by Sandro Bocci (de Paulis 2019).

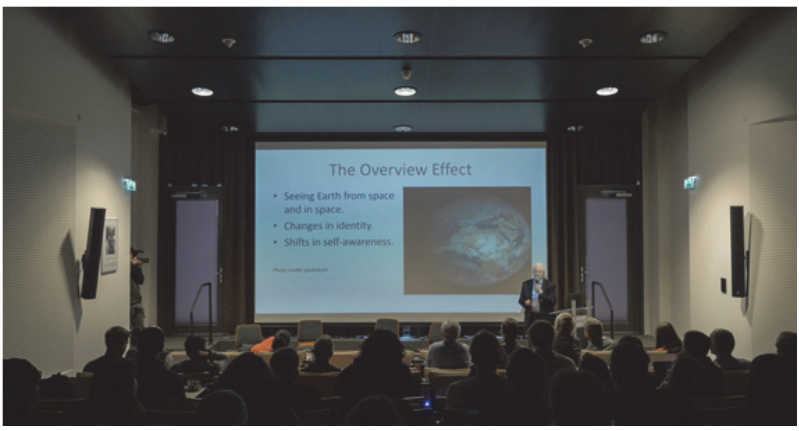

Figure 2: Frank White speaking about 'The Overview Effect' as part of a special event of COGITO in Space on 5 November 2018. Photo: Sandro Bocci.

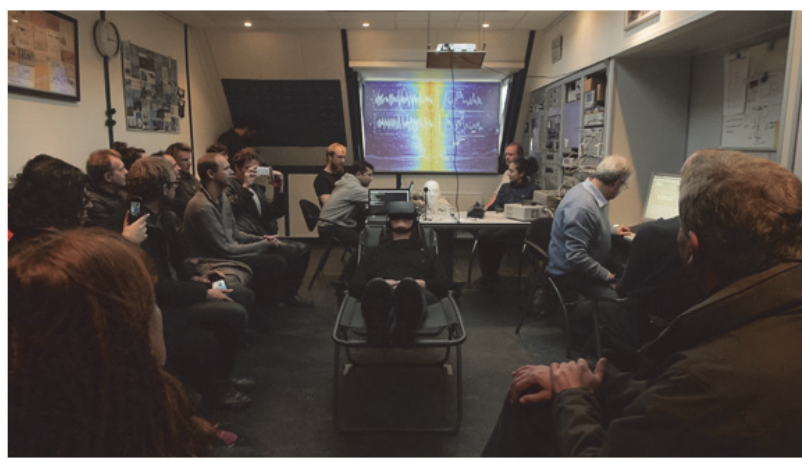

Figure 3: Performance of COGITO in Space inside the cabin of the Dwingeloo radio telescope. Photo: Sandro Bocci.

\section{REFERENCES}

Nagel, T. (2012) Mind \& Cosmos. Oxford University Press, New York.

de Paulis, D. (2017) Acoustic Space, Volume 16: Renewable Futures, Art, Science and Society in the Post-Media Age. RIXC, Riga.

de Paulis, D. (2019) Antennae: The Journal of Nature in Visual Culture.

Wall, M. (2013) Curiosity Rover Drills Into Mars Rock, Collects Sample - A Space First. https://www.space.com/19708-mars-rover-curiosityrock-drill-sample.html (retrieved 16 September 2018). 